\title{
On numerical techniques for solving the fractional logistic differential equation
}

\author{
Yves Yannick Yameni Noupoue ${ }^{1 *}$ (D, Yücel Tandoğdu ${ }^{1}$ and Muath Awadalla²
}

\author{
"Correspondence: \\ yves.yannick@emu.edu.tr \\ ${ }^{1}$ Department of Mathematics, \\ Eastern Mediterranean University, \\ Gazimagusa, North Cyprus, Turkey \\ Full list of author information is \\ available at the end of the article
}

\begin{abstract}
This paper studied the existence and uniqueness of the solution of the fractional logistic differential equation using Hadamard derivative and integral. Previous work has shown that there is not an exact solution to this fractional model. Hence several numerical approaches, such as generalized Euler's method (GEM), power series expansion (PSE) method, and Caputo-Fabrizio (CF) method, were used to compute the solution. The classical solution obtained from the first order non-linear differential equation was also considered to enable the comparison of error levels.
\end{abstract}

Keywords: Grunwald-Letnikov fractional derivative; Mathematical modeling; Non-linear systems; Numerical simulation; Fractional logistic equation

\section{Introduction}

The name fractional calculus stems from the fact that the order of derivatives and integrals are fractions rather than integers. Early work on fractional calculus dates back to the early nineteenth century [1]. Researchers initially concentrated on the proof of the existence and uniqueness of the solution to a fractional model [2-4]. Theory of fractional calculus has been discussed by many authors [5-7].

Recent research has concentrated on showing the advantages of fractional over classical calculus [4, 8-11]. In many cases, the fractional calculus has provided better results compared to those obtained via the classical approach. In general, the better performance of the fractional calculus becomes evident based on lower error levels produced during an estimation process $[8-10,12,13]$. Computational methods and numerical simulations successfully applied to several works, such as those found in [14-19], aimed to show the results proven theoretically or to iteratively compute the solution to problem whose analytic form is not explicit.

Evidence of fractional calculus being applicable to real life problems has gradually been proven by researchers in various branches of science. Such works are for instance found in biology $[8,11,12,20,21]$, in economy [9], and in physics $[8,10]$.

It is common in research to define a fractional calculus problem referring to its ordinary calculus counterpart when it exists. Such practice is often used in fractional differential equations. However, the structure of the solution to a fractional differential equation does not always correspond to its classical counterpart when it exists. Hence, an ordinary differential equation might have an exact solution whereas its fractional counterpart does not. The logistic differential equation [22] has an exact solution. However, West [23] proposed

(c) The Author(s) 2019. This article is distributed under the terms of the Creative Commons Attribution 4.0 International License (http://creativecommons.org/licenses/by/4.0/), which permits unrestricted use, distribution, and reproduction in any medium, provided you give appropriate credit to the original author(s) and the source, provide a link to the Creative Commons license, and indicate if changes were made. 
a power series referring to it as the exact solution to fractional logistic differential equation (FLDE). Subsequently Area et al. [24] claimed that West's proposal is valid only when the order of the derivative is one. However, the solution proposed by West was proven valid by D'Ovidio et al. [25], subject to changing the structure of the FLDE for what they called 'modified fractional logistic equation'. In [26] a numerical solution was proposed for FLDE based on Euler's method.

In this work the FLDE is investigated. Considering the fact that the FLDE is a non-linear equation whose exact solution does not exist, several numerical methods are used to compute its solution. Finally we compute the solution of FLDE by means of three numerical methods: the power series expansion (PSE) method also known as Letnikov method (LM) [27], the generalized Euler method (GEM) [28], and the Caputo-Fabrizio (CF) method [29]. The error rate of each of the mentioned methods is also computed for performance evaluation purpose. The introduction of the present work is followed by a preliminaries section in which some useful definitions are given, followed by a discussion on various numerical techniques used in the simulations. The fourth section is dedicated to the construction of the FLDE. The two last sections are respectively the simulation section and the concluding remarks.

\section{Preliminaries}

Some fractional calculus definitions and notation needed in the course of this work are discussed in this section.

Definition 2.1 ([30]) The Riemann-Liouville fractional integral of order $q>0$ of a function $g:[0,+\infty) \rightarrow \mathbb{R}$ is defined as

$$
\left({ }_{R L} I_{0^{+}}^{q} g\right)(t)=\frac{1}{\Gamma(q)} \int_{0}^{t}(t-s)^{q-1} g(s) d s,
$$

provided that the right-hand side of the integral is pointwise defined on $(0,+\infty)$ and $\Gamma$ is the gamma function $\Gamma(v)=\int_{0}^{\infty} e^{-t} t^{v-1} d t, \forall v>0$.

Definition 2.2 ([30]) The Riemann-Liouville fractional derivative of order $q>0$ of a function $g:[0,+\infty) \rightarrow \mathbb{R}$ is defined as

$$
\left({ }_{R L} D_{0^{+}}^{q} g\right)(t)=\frac{1}{\Gamma(n-q)}\left(\frac{d^{n}}{d t^{n}}\right) \int_{0}^{t}(t-s)^{n-q-1} g(s) d s,
$$

where $n-1 \leq q<n, n \in \mathbb{N}$.

Definition 2.3 ([30]) The Caputo derivative of order $q>0$ for a function $g:[0,+\infty] \rightarrow \mathbb{R}$ is defined as

$$
\left({ }_{C} D_{0^{+}}^{q} g\right)(t)= \begin{cases}\int_{0}^{t} \frac{(t-s)^{n-q-1} g^{(n)}(s)}{\Gamma(n-q)} d s, & n-1<q<n \in \mathbb{R} \\ g^{(n)}(t), & q \in \mathbb{N},\end{cases}
$$

where $n=[q]+1,[q]$ is the integer part of $q$. 
Definition 2.4 ([5]) Let $g$ be a continuous function and $n=\frac{t-a}{h}$, then the GrunwaldLetnikov (GL) fractional derivative of $g$ is given by

$$
\left({ }_{G L} D_{a^{+}}^{q} g\right)(t)=\lim _{h \rightarrow 0} \frac{1}{h^{q}} \sum_{j=0}^{\left[\frac{t-a}{h}\right]}(-1)^{j}\left(\begin{array}{l}
q \\
j
\end{array}\right) g(t-j h)
$$

where

$$
\left(\begin{array}{l}
q \\
j
\end{array}\right)=\frac{q !}{j !(q-j) !}=\frac{\Gamma(q+1)}{\Gamma(j+1) \Gamma(q-j+1)}, \quad \text { and } \quad\left(\begin{array}{l}
q \\
0
\end{array}\right)=1
$$

Definition 2.5 ([31]) Let $g \in H^{1}(a, b), b>a$, and $q \in[0,1]$. Then the new Caputo version of fractional derivative of $g$ is defined as

$$
\left({ }_{C F} D_{0}^{q} g\right)(t)=\frac{M(q)}{(1-q)} \int_{a}^{t} g^{\prime}(s) \exp \left[-\frac{q}{1-q}(t-s)\right] d s
$$

where $M(q)$ is the normalization function with $M(0)=M(1)=1$. If $g \notin H^{1}(a, b)$, then a new derivative, called Caputo-Fabrizio fractional derivative [31], is defined as

$$
\left({ }_{C F} D_{0}^{q} g\right)(t)=\frac{M(q)}{(1-q)} \int_{0}^{t} g^{\prime}(s) \exp \left[-\frac{q}{1-q}(t-s)\right] d s .
$$

The Caputo-Fabrizio fractional derivative has an advantage over other fractional derivatives of being a fractional derivative with non-singular kernel [31].

Definition 2.6 ([32]) The Hadamard fractional integral of order $q$ of a continuous function $g$ is defined as

$$
{ }^{H} I^{q} g(t)=\frac{1}{\Gamma(q)} \int_{a}^{t}\left(\ln \frac{t}{s}\right)^{q-1} \frac{g(s)}{s} d s, \quad q>0
$$

provided that the integral exists.

Definition 2.7 ([32]) The Hadamard fractional derivative of order $q>0$ of a continuous function $g:[a, \infty) \rightarrow \mathbb{R}$ is defined as

$$
{ }^{H} D^{q} g(t)=\delta^{n}\left({ }^{H} I^{q} g\right)(t)=\left(t \frac{d}{d t}\right)^{n} \frac{1}{\Gamma(n-q)} \int_{a}^{t}\left(\ln \frac{t}{s}\right)^{n-q-1} \frac{g(s)}{s} d s
$$

with $n-1<q<n, n=[q]+1 ; \delta=t\left(\frac{d}{d t}\right)$ and $[q]$ denotes the integer part of the real number $q$.

Lemma $2.8([32])$ Let $u, x \in C_{\delta}^{n}([a, T], \mathbb{R})$, where $C_{\delta}^{n}[a, T]=\left\{u, x:[a, T] \rightarrow \mathbb{R}: \delta^{(n-1)} u \in\right.$ $C[a, T]\}$, then

$$
{ }^{H} I^{q}\left({ }^{H} D^{q} u\right)(t)=u(t)-\sum_{j=1}^{n} c_{j}\left(\ln \frac{t}{a}\right)^{q-j} .
$$




\section{Numerical techniques for solving non-linear differential equations}

We discuss in this section some general numerical methods often used to find the numerical solution to non-linear fractional differential equations. These methods are later applied to the FLDE.

\subsection{The generalized Euler's method (GEM)}

GEM is discussed here. The method was introduced by Odibat et al. [28]. It is derived from the known Euler's method for solving differential equations. Consider the fractional order non-linear differential equation given by

$$
\left({ }_{C} D_{0^{+}}^{q} x\right)(t)=g(t, x(t)), \quad x(0)=0,
$$

where the fractional order of derivative $q \in(0,1]$ and $t>0$. Moreover, we assume that the following functions $x(t),{ }_{C} D_{0^{+}}^{q} x(t)$, and ${ }_{C} D_{0^{+}}^{2 q} x(t)$ are continuous on the closed interval $[0, T]$. In order to find the numerical solution to the problem defined by Eq. (3.1) over the interval $[0, T]$, a discretization of $[0, T]$ into $k$ sub-intervals $\left[t_{j}, t_{j+1}\right]$ of equal width $h=T / k$ is required. The set of points $\left\{t_{j}, x\left(t_{j}\right)\right\}$ is also used in the approximation process. The GEM approximated solution is given by

$$
x\left(t_{j+1}\right)=x\left(t_{j}\right)+\frac{h^{q}}{\Gamma(q+1)} g\left(t_{j}, x\left(t_{j}\right)\right), \quad j=0,1, \ldots, k-1,
$$

where the node $t_{j}=j h, j=1,2, \ldots, k$.

\subsection{The Grünwald-Letnikov method (GL) or power series expansion (PSE)}

Grünwald provided a numerical approach for solving non-linear differential equation. This approach is discussed in detail in [27].

Definition 3.1 ([27]) The explicit fractional numerical approximation formula of $q$ th derivative at the points $k h(k=1,2, \ldots)$ in the Grünwald-Letnikov sense has the following form:

$$
\left(k-L_{m} / h\right)\left({ }_{G L} D_{t_{k}}^{q} g\right)(t) \approx \frac{1}{h^{q}} \sum_{j=0}^{k}(-1)^{j}\left(\begin{array}{l}
q \\
j
\end{array}\right) g\left(t_{k-j}\right),
$$

where $L_{m}$ is the memory length; $t_{k}=k h$; the time-space step of iteration is $h$ and $(-1)^{j}\left(\begin{array}{l}q \\ j\end{array}\right)$ are referred to as binomial coefficients. For computational issue, the binomial coefficients are usually denoted by $c_{j}^{(q)}(j=0,1, \ldots)$ and computed as follows:

$$
\left\{\begin{array}{l}
c_{0}^{(q)}=1, \\
c_{j}^{(q)}=\left(1-\frac{1+q}{j}\right) c_{j-1}^{(q)} .
\end{array}\right.
$$

Consider a non-linear fractional differential equation, where the fractional derivative is taken in the Grünwald-Letnikov sense, with initial condition, defined by

$$
\left({ }_{G L} D_{a^{+}}^{q} u\right)(t)=g(u(t), t)
$$


the numerical solution to the problem stated by Eq. (3.4) is given by

$$
u\left(t_{k}\right)=g\left(u\left(t_{k}\right), t_{k}\right) h^{q}-\sum_{j=1}^{k} c_{j}^{(q)} u\left(t_{k-j}\right)
$$

\subsection{The Caputo-Fabrizio method (CF)}

Consider a non-linear fractional differential equation with initial condition, where the fractional derivative is taken in the Caputo-Fabrizio sense, defined by

$$
\left({ }_{C F} D_{0}^{q} u\right)(t)=g(t, u(t)), \quad u(0)=u_{0}
$$

The numerical solution to the problem defined by Eq. (3.6) is built based on the AdamBasforth method as follows (see $[29,33])$ :

$$
u_{n+1}=u_{n}+\left(\frac{1-q}{M(q)}+\frac{3 q h}{2 M(q)}\right) g\left(t_{n}, u_{n}\right)+\left(\frac{1-q}{M(q)}+\frac{q h}{2 M(q)}\right) g\left(t_{n-1}, u_{n-1}\right) .
$$

\section{Fractional logistic differential equation}

In this section, the general form of the numerical solution of FLDE is discussed. The logistic model is used for population growth modeling. Given a population with proliferation capability, with an initial size of $P_{0}$, one would obviously think that the size of the population will infinitely increase when the time approaches infinity. However, it has been proven by Malthus (see [34]) that a population size tends to stabilize when the time approaches infinity. The growth trend obtained from the Malthusian theory is what we call the logistic growth model. The model is defined classically by the following differential equation:

$$
\frac{d N(t)}{d t}=r N(t)\left(1-\frac{N(t)}{K}\right)
$$

where the initial population size is $N(0)=N_{0}, r$ is called the growth rate; $K$ is called the carrying capacity. It is the maximum value that the population size can reach. $N(t)$ is the population size at time $t$.

The exact solution of the classical logistic differential equation defined by Eq. (4.1) is given by

$$
N_{c}(t)=\frac{K}{1+\left(\frac{K-N_{0}}{N_{0}}\right) e^{-r t}} .
$$

Without loss of generality, Eq. (4.1) can be converted to FLDE based on the Hadamard fractional derivative as follows:

$$
\left({ }^{H} D_{0}^{q} N\right)(t)=r N(t)\left(1-\frac{N(t)}{K}\right), \quad N(a)=N_{a}<\infty .
$$

\subsection{Existence and uniqueness of the solution of fractional logistic equation}

In this section we prove the existence and uniqueness of the solution of the FLDE. Without loss of generality, the Hadamard fractional derivative and integral symbols are used. 
Applying the operator ${ }^{H} I^{r}$ to Eq. (4.3), we obtain the following:

$$
N(t)-c_{1}\left(\ln \frac{t}{a}\right)^{q-1}={ }^{H} I^{q}\left(r N\left(1-\frac{N}{K}\right)\right) .
$$

For simplicity, the following kernel function is chosen:

$$
Q(t, N(t))=r N\left(1-\frac{N}{K}\right)
$$

Note that the initial value condition implies $c_{1}=N_{a}$.

Let $H=C([a, T], \mathbb{R})$ denote the Banach space of all continuous functions from $[a, T]$ to $\mathbb{R}$, we identify the operator $E: H \rightarrow H$ endowed with the norm $\|N\|=\sup _{a \leq t \leq T}|N(t)|$, then

$$
(E N)(t)=N_{a}\left(\ln \frac{t}{a}\right)^{q-1}+\frac{1}{\Gamma(q)} \int_{a}^{t}\left(\ln \frac{t}{s}\right)^{q-1} \frac{Q(s, N(s))}{s} d s .
$$

\subsubsection{Existence of solution}

Using the setting above, the existence of the solution is stated and proven by Theorem 4.1 as follows.

Theorem 4.1 Let $Q:[a, T] \times \mathbb{R} \rightarrow \mathbb{R}$ be a continuous function such that the following assumptions hold:

$\left(A_{1}\right) \exists N_{Q}>0$ such that $\left|Q\left(t, N_{1}\right)-Q\left(t, N_{2}\right)\right| \leq N_{Q}\left|N_{1}-N_{2}\right|, \forall t \in[a, T], \forall N_{1}, N_{2} \in \mathbb{R}$.

$\left(A_{2}\right)|Q(t, N)| \leq y(t), \forall(t, N) \in[0, T] \times \mathbb{R}$, where, $y \in C\left([a, T], \mathbb{R}^{+}\right)$with $\sup _{a \leq t \leq T}|y(t)|=$ $\|y\|$.

In addition, it is assumed that $\frac{N_{Q}}{\Gamma(q+1)}\left(\ln \frac{T}{a}\right)^{q-1}<1$, then there is at least one solution for the initial value problem given by Eq. (4.3).

Proof of Theorem 4.1 Consider the close set $B_{\lambda}=\{N \in H,\|N\| \leq \lambda\}$ with

$$
\lambda \geq N_{a}\left(\ln \frac{T}{a}\right)^{q-1}+\frac{1}{\Gamma(q+1)}\left(\ln \frac{T}{a}\right)^{q}\|y\|
$$

We define the operators $E_{1}$ and $E_{2}$ on $B_{\lambda}$ as

$$
\left(E_{1} N\right)(t)=N_{a}\left(\ln \frac{t}{a}\right)^{q-1} ; \quad\left(E_{2} N\right)(t)=\frac{1}{\Gamma(q)} \int_{a}^{t}\left(\ln \frac{t}{s}\right)^{q-1} \frac{Q(s, N(s))}{s} d s .
$$

For $N_{1}, N_{2} \in B_{\lambda}$, then $\left\|E_{1} N_{1}+E_{2} N_{2}\right\| \leq N_{a}\left(\ln \frac{T}{a}\right)^{q-1}+\frac{1}{\Gamma(q+1)}\left(\ln \frac{T}{a}\right)^{q}\|y\| \leq \lambda$, thus $E_{1} N_{1}+$ $E_{2} N_{2} \in B_{\lambda}$.

Next, we show that $E_{2}$ is a contraction, $\forall t \in[a, T], \forall N_{1}, N_{2} \in B_{\lambda}$, we have

$$
\begin{aligned}
\left|E_{2} N_{1}(t)-E_{2} N_{2}(t)\right| & =\left|\int_{a}^{t}\left(\ln \frac{t}{s}\right)^{q-1} \frac{Q\left(s, N_{1}(s)\right)}{s} d s-\int_{a}^{t}\left(\ln \frac{t}{s}\right)^{q-1} \frac{Q\left(s, N_{2}(s)\right)}{s} d s\right| \\
& \leq \frac{1}{\Gamma(q)} \int_{a}^{t}\left(\ln \frac{t}{s}\right)^{q-1}\left|Q\left(s, N_{1}(s)\right)-Q\left(s, N_{2}(s)\right)\right| \frac{d s}{s}
\end{aligned}
$$


then

$$
\begin{aligned}
\left\|E_{2} N_{1}+E_{2} N_{2}\right\| & \leq \frac{1}{\Gamma(q+1)} N_{Q}\left(\ln \frac{T}{a}\right)^{q-1}\left\|N_{1}-N_{2}\right\| \\
& \leq\left\|N_{1}-N_{2}\right\|,
\end{aligned}
$$

which implies that $E_{2}$ is a contraction.

In addition, the operator $E_{1}$ is continuous as a result of the continuity of $N$. Moreover, $E_{1}$ is uniformly bounded as

$$
\left\|E_{1} N\right\| \leq N_{a}\left(\ln \frac{T}{a}\right)^{q-1}
$$

Now it will be shown that the operator $E_{1}$ is compact for $t_{1}, t_{2} \in[a, T]\left(t_{1}<t_{2}\right)$, then

$$
\left\|\left(E_{1} N\right)\left(t_{2}\right)-\left(E_{1} N\right)\left(t_{1}\right)\right\| \leq N_{a}\left(\left|\left(\ln \frac{t_{2}}{a}\right)^{q-1}-\left(\ln \frac{t_{1}}{a}\right)^{q-1}\right|\right) .
$$

The right-hand side of the above inequality approaches zero as $t_{1} \rightarrow t_{2}$. Note that $\left\|\left(E_{1} N\right)\left(t_{2}\right)-\left(E_{1} N\right)\left(t_{1}\right)\right\|$ is independent of $N$ implies that $E_{1}$ is relatively compact, by Arzela-Ascoli theorem we conclude that $E_{1}$ is compact on $B_{\lambda}$. Hence, the existence of the solution of the initial value problem given by Eq. (4.3) holds by Krasnoselskii's fixed point theorem.

\subsubsection{Uniqueness of solution}

Theorem 4.2 Let $Q:[a, T] \times \mathbb{R} \rightarrow \mathbb{R}$ be a continuous function satisfying $\left(A_{1}\right)$ and assume that $\left(\frac{1}{\Gamma(q+1)}\left(\ln \frac{T}{a}\right)^{q}\right) N_{Q}<1$, then the initial value problem given by $E q$. (4.3) has a unique solution.

Proof Theorem 4.2 Consider the close set $B_{\lambda}=\{N \in H,\|N\| \leq \lambda\}$ with

$$
\lambda \geq \frac{N_{a}\left(\ln \frac{T}{a}\right)^{q-1}+M\left(\frac{1}{\Gamma(q+1)}\left(\ln \frac{T}{a}\right)^{q}\right)}{1-N_{Q}\left(\frac{1}{\Gamma(q+1)}\left(\ln \frac{T}{a}\right)^{q}\right)}, \quad \text { where } M=\sup _{a \leq t \leq T}|Q(t, 0)| .
$$

First we show that $E B_{\lambda} \subset B_{\lambda}$. For any $N \in B_{\lambda}, \forall t \in[a, T]$,

$$
|(E N)(t)| \leq N_{a}\left(\ln \frac{t}{a}\right)^{q-1}+\frac{1}{\Gamma(q)} \int_{a}^{t}\left(\ln \frac{t}{s}\right)^{q-1} \frac{|Q(s, N(s))|}{s} d s
$$

but

$$
\begin{aligned}
|Q(t, N(t))| & =|Q(t, N(t))-Q(t, 0)+Q(t, 0)| \\
& \leq|Q(t, N(t))-Q(t, 0)|+|Q(t, 0)| \\
& \leq N_{Q}\|N\|+M \\
& \leq N_{Q} \lambda+M,
\end{aligned}
$$


then

$$
\begin{aligned}
\|E N\| & \leq N_{a}\left(\ln \frac{T}{a}\right)^{q-1}+\left(\frac{1}{\Gamma(q+1)}\left(\ln \frac{T}{a}\right)^{q}\right)\left(N_{Q} \lambda+M\right) \\
& \leq \lambda
\end{aligned}
$$

which implies that $E N \in B_{\lambda}, \forall N \in B_{\lambda}$, that is, $E B_{\lambda} \subset B_{\lambda}$. Then it is sufficient to show that the operator $E$ is a contraction.

$$
\begin{aligned}
& \forall N_{1}, N_{2} \in H \\
& \left|E N_{1}(t)-E N_{2}(t)\right| \leq \frac{1}{\Gamma(q)} \int_{a}^{t}\left(\ln \frac{t}{s}\right)^{q-1}\left|Q\left(s, N_{1}(s)\right)-Q\left(s, N_{2}(s)\right)\right| \frac{d s}{s},
\end{aligned}
$$

which implies that

$$
\begin{aligned}
\left\|E N_{1}-E N_{2}\right\| & \leq\left(\frac{1}{\Gamma(q+1)}\left(\ln \frac{T}{a}\right)^{q}\right) N_{Q}\left\|N_{1}-N_{2}\right\| \\
& \leq\left\|N_{1}-N_{2}\right\| .
\end{aligned}
$$

So $E$ is a contraction. By Banach's contraction mapping theorem, the initial value problem given by Eq. (4.3) has a unique solution on $[a, T]$.

\section{Simulation studies}

In the literature review it has become evident that no one has so far proposed a valid exact form of solution to FLDE. In Sect. 4 the existence and uniqueness of the solution of FLDE is proven. Since there is no exact solution to the FLDE problem, approximate solutions are found using the GEM, PSE, and CF methods.

Let $q \in(0,1) \cup(1,2)$ be the fractional order of derivative, the numerical solutions of the FLDE using GEM, PSE, and CF are respectively given by

$$
\begin{aligned}
N_{G E M}\left(t_{j+1}\right)= & N\left(t_{j}\right)+\frac{h^{q}}{\Gamma(q+1)} r N\left(t_{j}\right)\left(1-\frac{N\left(t_{j}\right)}{K}\right), \quad j=0,1, \ldots k-1, \\
N_{P S E}\left(t_{k}\right)= & r N\left(t_{k}\right)\left(1-\frac{N\left(t_{k}\right)}{K}\right) h^{q}-\sum_{j=1}^{k} c_{j}^{(q)} N\left(t_{k-j}\right), \\
N_{C F}\left(t_{n+1}\right)= & N\left(t_{n}\right)+\left(\frac{1-q}{M(q)}+\frac{3 q h}{2 M(q)}\right) N\left(t_{n}\right)\left(1-\frac{N\left(t_{n}\right)}{K}\right) \\
& +\left(\frac{1-q}{M(q)}+\frac{q h}{2 M(q)}\right) N\left(t_{n-1}\right)\left(1-\frac{N\left(t_{n-1}\right)}{K}\right) .
\end{aligned}
$$

In order to evaluate the performance of these methods, the error rate between the true data values and the values computed using fractional differential equations is computed using the formula below [35]:

$$
E R=\sqrt{\frac{\sum_{i=0}^{n}\left(y_{i}(t)-\hat{y}_{i}(t)\right)^{2}}{n}} / \sqrt{\frac{\sum_{i=0}^{n}\left(y_{i}(t)\right)^{2}}{n}},
$$


where $y_{i}(t)$ is the true value at time $t ; \hat{y}_{i}(t)$ is the estimate value at time $t ; n$ is the sample datasize.

Experimental data comes from the study of the annual growth rate of helianthus plant. The data is retrieved from [36]. The height of the plants measured at a constant spacing time of 7 days is given in centimeters. Twelve measurements were considered, ranging from the seventh day to the eighty-fourth day of the plant life. The mean values of the heights are considered.

Considering Eq. (4.2) that gives the solution of the classical approach, the growth rate $r=0.0760$ and the carrying capacity $K=267.5301$ for this data set are obtained through a non-linear optimization tool. The 'lsqcurvefit' Matlab command was used in this case. The classical approach Eq. (4.2) produced an error rate $E R=0.0320$ that is 3.2\%. The graph of the classical solution alongside the true data is given in Fig. 1.

Recalling Eq. (5.1) and Eq. (5.2) for the GEM and PSE numerical solutions respectively, it was decided to determine the value of the fractional order of derivative $q$ for which the error rate $E R$ is minimized. The interval $[0.9,1.2]$ was iteratively covered by all the possible $q$ values using $10^{-3}$ steps. Both GEM and PSE minimized $E R$ for $q=1$ as shown in Fig. 2.

$E R$ is minimized at $q=1$ for both GEM and PSE, that is, when both methods coincide with the classical method. At $q=1$, both methods have $E R=0.0320$ or $3.2 \%$, which is the same as the error rate obtained for the classical approach. The graphs in Fig. 3 show the PSE and GEM data for $q=1$.

In order to show the behavior of the results produced by GEM and PSE for different values of $q$, let $q=0.9$. Then the GEM method produced $E R=0.2954$, that is, $29.54 \%$, whereas the PSE produced $E R=0.4146$ or $41.46 \%$. Figure 2 clearly illustrates variation of the error rate $E R$ with respect to $q$ values. The graphs obtained for GEM and PSE methods when $q=0.9$ are shown in Fig. 4. In conformity with Fig. 2, where error rates for GEM and Letnikov are about 0.29 and 0.41 , respectively, deviation of the estimated values from the true ones is greater in the Letnikov case as seen in Fig. 4.

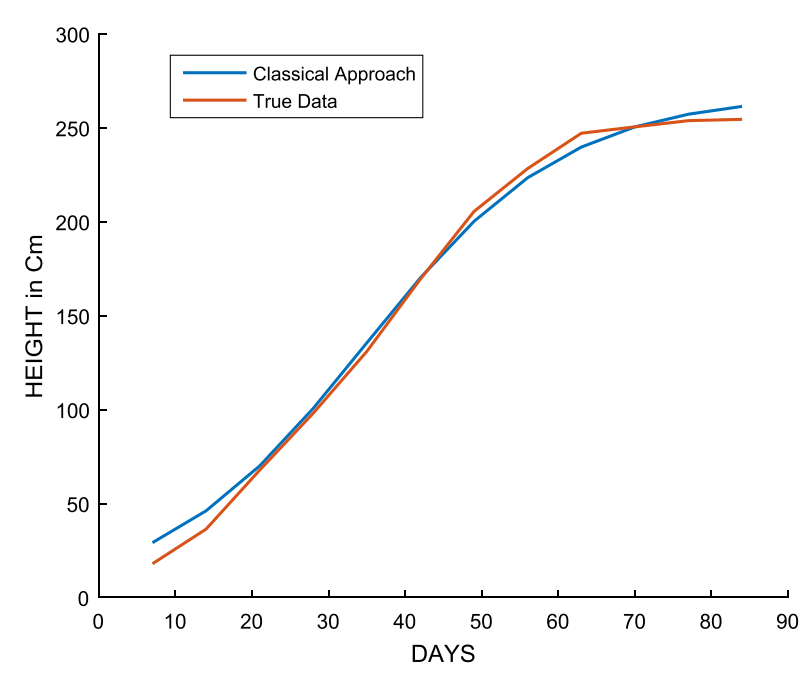

Figure 1 Classical Method 


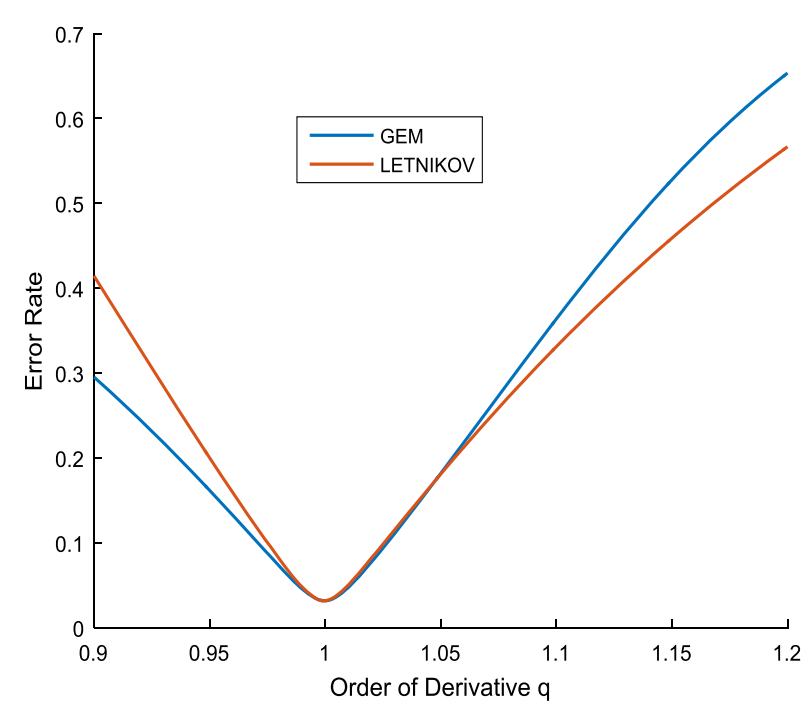

Figure 2 GEM and PSE error rate versus $q$ values

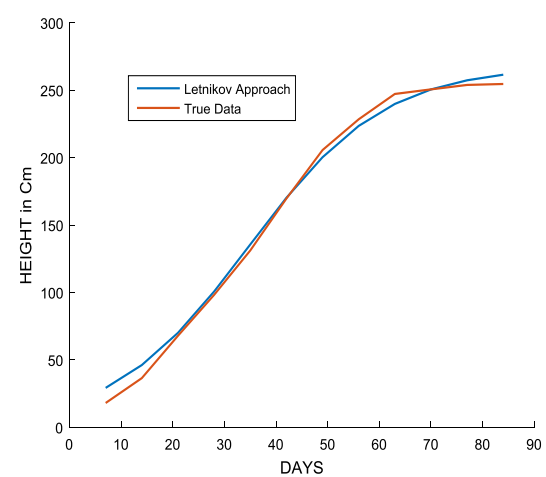

(a)

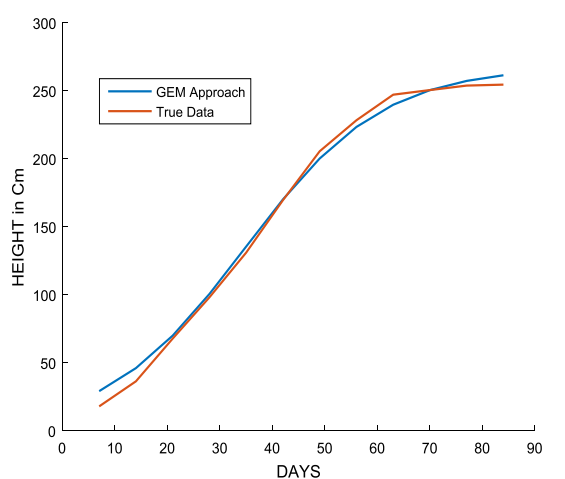

(b)

Figure 3 Graphs of (a) PSE with $q=1$ and (b) GEM for $q=1$

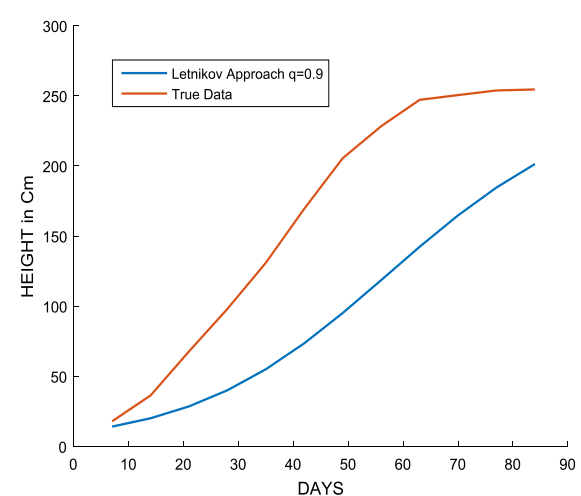

(a)

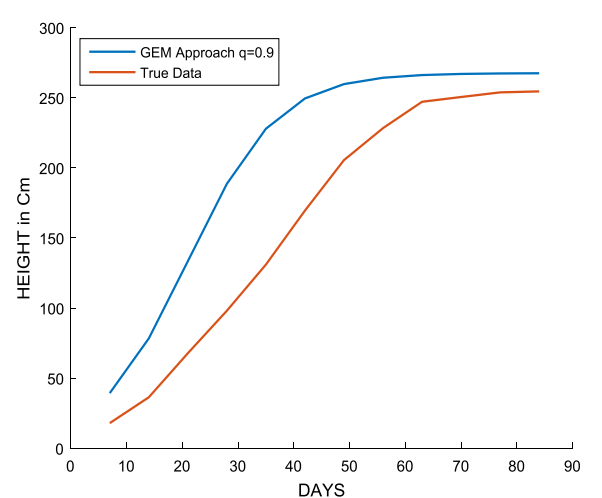

(b)

Figure 4 Graphs of (a) PSE with $q=0.9$ and (b) GEM for $q=0.9$ 


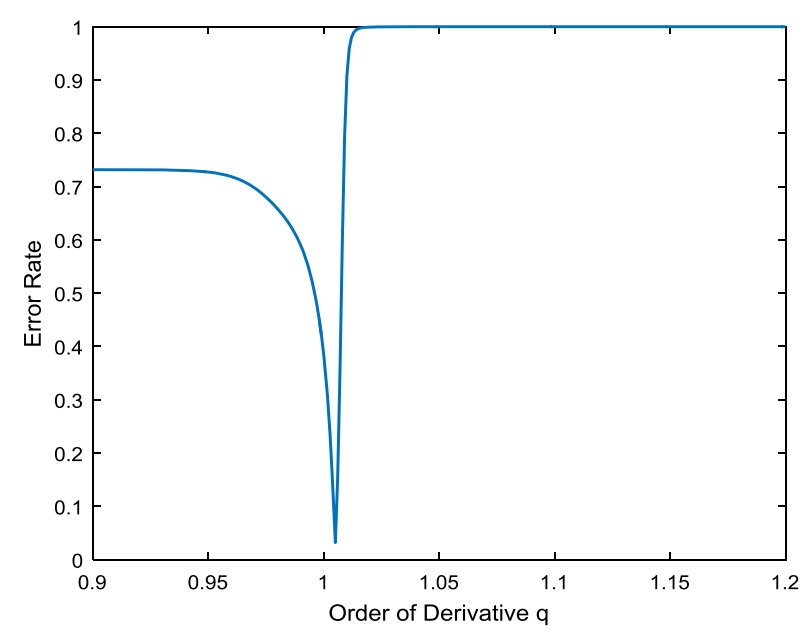

Figure 5 CF's error rate versus q values

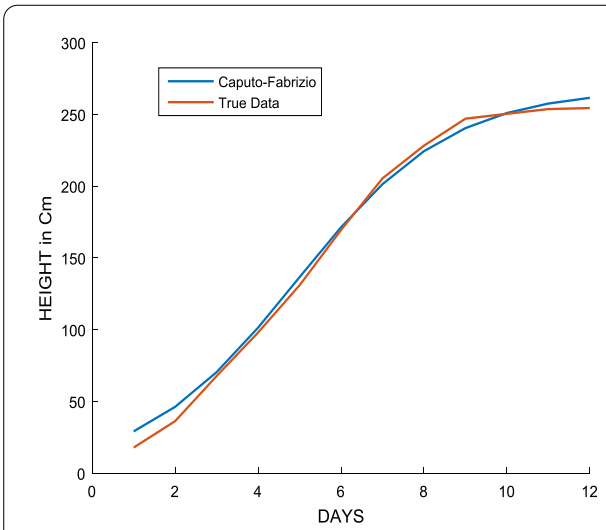

(a)

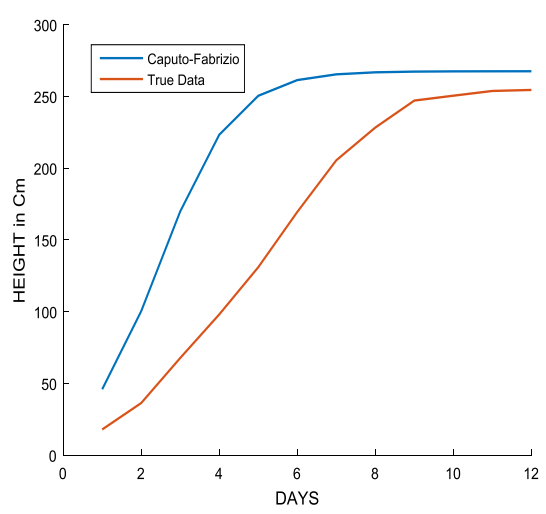

(b)

Figure 6 CF 's graph (a) for $q=1.005$ and $(\mathbf{b})$ for $q=1$

To find the value of $q$ for which the CF method, Eq. (5.3), minimizes the error rate $E R$, $q$ values ranging between 0.9 and 1.2 with increments $10^{-3}$ were used. Minimum error ( $E R=0.0321$ or $3.21 \%$ ) was obtained when $q=1.005$ as seen in Fig. 5 .

The graphs of the simulation obtained for the optimal value of $q=1.005$, for the value of $q=1$ together with the original data are shown in Fig. 6. The CF method produced $E R=0.3824$ that is $38.24 \%$ for the value of $q=1$, and much lower error $(E R=0.0321$ or $3.21 \%)$ when $q=1.005$.

\section{Conclusion}

The FLDE is studied, the existence and uniqueness of a solution are proposed and proved by means of the Hadamard fractional derivative and integral formula. The proposed solution is shown to be applicable in practice by using a data set and employing three numerical approaches, namely the GEM, PSE, and CF.

The values of the fractional derivative of order $q$, for which each of the method produces a minimum error rate $E R$, were found iteratively. It appeared that the GEM and PSE 
methods produce a common minimum error rate of $E R=3.2 \%$ for a value of $q=1$. The CF method produces the same minimum error rate of $E R=3.2 \%$ for a value of $q=1.005$.

It is worth mentioning that the fractional derivative of order $q=1$ coincides with the classical approach of the solution. Through the application problem it has become evident that the CF method has performed better in solving the FLDE, by producing a minimum error rate for $q$ value different from 1 .

\section{Acknowledgements}

The authors thank the reviewers for their useful comments, which led to the improvement of the original manuscript.

\section{Funding}

This research received no specific grant from any funding agency in the public, commercial, or not-for-profit sectors. The work was not performed as part of the employment of the authors.

\section{Competing interests}

The authors declare no conflict of interests.

\section{Authors' contributions}

Supervision, YT; writing - original draft, MA and YYYN. All authors read and approved the final manuscript.

\section{Author details}

'Department of Mathematics, Eastern Mediterranean University, Gazimagusa, North Cyprus, Turkey. ${ }^{2}$ Department of Energy Engineering, Girne American University, Kyrenia, North Cyprus, Turkey.

\section{Publisher's Note}

Springer Nature remains neutral with regard to jurisdictional claims in published maps and institutional affiliations.

Received: 15 December 2018 Accepted: 5 March 2019 Published online: 14 March 2019

\section{References}

1. Debnath, L.: A brief historical introduction to fractional calculus. Int. J. Math. Educ. Sci. Technol. 35(4), 487-501 (2004)

2. Mahmudov, N.I., Awadalla, M., Abuassba, K.: Nonlinear sequential fractional differential equations (2017)

3. Mahmudov, N.I., Awadalla, M., Abuassba, K.: Hadamard and Caputo-Hadamard FDE's with Three Point Integral Boundary Conditions (2017)

4. Inc, M., Yusuf, A., Aliyu, A.I., Baleanu, D.: Investigation of the logarithmic-KdV equation involving Mittag-Leffler type kernel with Atangana-Baleanu derivative. Phys. A, Stat. Mech. Appl. 506, 520-531 (2018)

5. Podlubny, I.: Fractional Differential Equations: An Introduction to Fractional Derivatives, Fractional Differential Equations, to Methods of Their Solution and Some of Their Applications, vol. 198. Elsevier, Amsterdam (1998)

6. Sabatier, J., Agrawal, O.P., Machado, J.T.: Advances in Fractional Calculus, vol. 4. Springer, Dordrecht (2007)

7. Oldham, K., Spanier, J.: The Fractional Calculus Theory and Applications of Differentiation and Integration to Arbitrary Order. vol. 111. Elsevier, Amsterdam (1974)

8. Almeida, R., Bastos, N.R., Monteiro, M.T.T.: Modeling some real phenomena by fractional differential equations. Math Methods Appl. Sci. 39(16), 4846-4855 (2016)

9. Almeida, R., Malinowska, A.B., Monteiro, M.T.T.: Fractional differential equations with a Caputo derivative with respect to a kernel function and their applications. Math. Methods Appl. Sci. 41(1), 336-352 (2018)

10. Awadalla, M., Yameni, Y.Y.: Modeling exponential growth and exponential decay real phenomena by $\Psi$-Caputo fractional derivative. J. Adv. Math. Comput. Sci. 28(2), 2456-9968 (2018). https://doi.org/10.9734/JAMCS/2018/43054

11. Yusuf, A., Qureshi, S., Inc, M., Aliyu, A.I., Baleanu, D., Shaikh, A.A.: Two-strain epidemic model involving fractional derivative with Mittag-Leffler kernel. Chaos, Interdiscip. J. Nonlinear Sci. 28(12), 123121 (2018)

12. Qureshi, S., Yusuf, A., Shaikh, A.A., Inc, M., Baleanu, D.: Fractional modeling of blood ethanol concentration system with real data application. Chaos, Interdiscip. J. Nonlinear Sci. 29(1), 013143 (2019)

13. Yusuf, A., Inc, M., Aliyu, A.I., Baleanu, D.: Efficiency of the new fractional derivative with nonsingular Mittag-Leffler kernel to some nonlinear partial differential equations. Chaos Solitons Fractals 116, 220-226 (2018)

14. Ullah, A., Shah, K.: Numerical analysis of Lane Emden-Fowler equations. J. Taibah Univ. Sci. 12(2), 180-185 (2018)

15. Shah, K., Ali, A., Khan, R.A.: Numerical solutions of fractional order system of Bagley-Torvik equation using operational matrices. Sindh Univ. Res. J. (Sci. Ser.) 47(4), 757-762 (2015)

16. Khalil, H., Shah, K., Khan, R.A.: Approximate solution of boundary value problems using shifted Legendre polynomials. Appl. Comput. Math. 16(3), 269-285 (2017)

17. Inc, M., Abdel-Gawad, H.I., Tantawy, M., Yusuf, A.: On multiple soliton similariton-pair solutions, conservation laws via multiplier and stability analysis for the Whitham-Broer-Kaup equations in weakly dispersive media. Math. Methods Appl. Sci.

18. Abdel-Gawad, H.l., Tantawy, M., Inc, M., Yusuf, A.: On multi-fusion solitons induced by inelastic collision for quasi-periodic propagation with nonlinear refractive index and stability analysis. Mod. Phys. Lett. B 32(29), 1850353 (2018)

19. Ghanbari, B., Yusuf, A., Baleanu, D.: The new exact solitary wave solutions and stability analysis for the \$-dimensional Zakharov-Kuznetsov equation. Adv. Differ. Equ. 2019(1), 49 (2019) 
20. Bushnaq, S., Khan, S.A., Shah, K., Zaman, G.: Existence theory of HIV-1 infection model by using arbitrary order derivative of without singular kernel type. J. Math. Anal. 9(1), 16-28 (2018)

21. Aliyu, A.I., Inc, M., Yusuf, A., Baleanu, D.: A fractional model of vertical transmission and cure of vector-borne diseases pertaining to the Atangana-Baleanu fractional derivatives. Chaos Solitons Fractals 116, 268-277 (2018)

22. Petropoulou, E:: A discrete equivalent of the logistic equation. Adv. Differ. Equ. 2010(1), 457073 (2010)

23. West, B.J.: Exact solution to fractional logistic equation. Phys. A, Stat. Mech. Appl. 429, 103-108 (2015)

24. Area, I., Losada, J., Nieto, J.J.: A note on the fractional logistic equation. Phys. A, Stat. Mech. Appl. 444, 182-187 (2016)

25. D'Ovidio, M., Loreti, P., Ahrabi, S.S.: Modified fractional logistic equation. Phys. A, Stat. Mech. Appl. 505, 818-824 (2018)

26. Vivek, D., Kanagarajan, K., Harikrishnan, S.: Numerical solution of fractional-order logistic equations by fractional Euler's method (2013)

27. Petráš, I.: Fractional-Order Nonlinear Systems: Modeling, Analysis and Simulation. Springer, Berlin (2011)

28. Odibat, Z.M., Momani, S.: An algorithm for the numerical solution of differential equations of fractional order. J. Appl. Math. Inform. 26(1-2), 15-27 (2008)

29. Atangana, A., Owolabi, K.M.: New numerical approach for fractional differential equations. Math. Model. Nat. Phenom. 13(1), $3(2018)$

30. Diethelm, K.: The Analysis of Fractional Differential Equations: An Application-Oriented Exposition Using Differential Operators of Caputo Type. Springer, Berlin (2010)

31. Caputo, M., Fabrizio, M.: A new definition of fractional derivative without singular kernel. Prog. Fract. Differ. Appl. 1(2), 1-13 (2015)

32. Pooseh, S., Almeida, R., Torres, D.F.: Expansion formulas in terms of integer-order derivatives for the Hadamard fractional integral and derivative. Numer. Funct. Anal. Optim. 33(3), 301-319 (2012)

33. Bastos, N.R.: Calculus of variations involving Caputo-Fabrizio fractional differentiation. Statistics 2310, 5070 (2018)

34. Malthus, T.R.: An essay on the principle of population: or, A view of its past and present effects on human happiness. Reeves \& Turner. (1888)

35. Tandoğdu, Y., Erbilen, M.: Imputing missing values using support variables with application to barley grain yield. J. Agric. Sci. Technol. 20(4), 829-839 (2018)

36. Reed, H.S., Holland, R.H.: The growth rate of an annual plant Helianthus. Proc. Natl. Acad. Sci. 5(4), 135-144 (1919)

\section{Submit your manuscript to a SpringerOpen ${ }^{\circ}$ journal and benefit from:}

- Convenient online submission

- Rigorous peer review

- Open access: articles freely available online

- High visibility within the field

- Retaining the copyright to your article

Submit your next manuscript at $\boldsymbol{\nabla}$ springeropen.com 